\title{
Genomic comparison of closely related Giant Viruses supports an accordion-like model of evolution
}

\author{
Jonathan Filée * \\ Laboratoire Evolution, Génome, Comportement, Ecologie, Centre National de la Recherche Scientifique UMR 9191, IRD \\ UMR 247, Université Paris-Saclay, Gif-sur-Yvette, France
}

OPEN ACCESS

Edited by:

Bernard La Scola,

Aix Marseille Université, France

Reviewed by:

Dahlene N. Fusco,

Massachusetts General Hospital, USA

Philippe Colson,

Aix-Marseille Université, France

*Correspondence: Jonathan Filée

Laboratoire Evolution, Génome,

Comportement, Ecologie. Centre National de la Recherche Scientifique UMR 9191, IRD UMR 247, Université

Paris-Saclay, Gif-sur-Yvette, France jonathan.filee@legs.cnrs-gif.fr

Specialty section: This article was submitted to Virology,

a section of the journal

Frontiers in Microbiology

Received: 24 March 2015

Accepted: 29 May 2015

Published: 16 June 2015

Citation:

Filée J (2015) Genomic comparison of closely related Giant Viruses supports an accordion-like model of evolution.

Front. Microbiol. 6:593.

doi: 10.3389/fmich.2015.00593
Genome gigantism occurs so far in Phycodnaviridae and Mimiviridae (order Megavirales). Origin and evolution of these Giant Viruses (GVs) remain open questions. Interestingly, availability of a collection of closely related GV genomes enabling genomic comparisons offer the opportunity to better understand the different evolutionary forces acting on these genomes. Whole genome alignment for five groups of viruses belonging to the Mimiviridae and Phycodnaviridae families show that there is no trend of genome expansion or general tendency of genome contraction. Instead, GV genomes accumulated genomic mutations over the time with gene gains compensating the different losses. In addition, each lineage displays specific patterns of genome evolution. Mimiviridae (megaviruses and mimiviruses) and Chlorella Phycodnaviruses evolved mainly by duplications and losses of genes belonging to large paralogous families (including movements of diverse mobiles genetic elements), whereas Micromonas and Ostreococcus Phycodnaviruses derive most of their genetic novelties thought lateral gene transfers. Taken together, these data support an accordion-like model of evolution in which GV genomes have undergone successive steps of gene gain and gene loss, accrediting the hypothesis that genome gigantism appears early, before the diversification of the different GV lineages.

Keywords: giant virus, genome, lateral gene transfer, duplication, evolution, mobile genetic elements

\section{Introduction}

The order Megavirales (formerly NCLDVs: Nucleo Cytoplasmic Large DNA Viruses) comprises very diverse double strand DNA viruses infecting a wide range of eukaryotic hosts, including vertebrates, protists, and unicellular alga (Colson et al., 2013). This group is supposed to be monophyletic on the basis of a conserved set of 25-50 genes encoding mainly structural and informational proteins (Iyer et al., 2006; Yutin et al., 2013). Seven different families compose the order: Poxviridae, Asfarviridae, Iridoviridae, Ascoviridae, Phycodnaviridae, Mimiviridae, and Marseilleviridae. Among them, two families display important variations in term of genome size: the Mimiviridae ranging from 371 to $1259 \mathrm{~kb}$ (Arslan et al., 2011; Moniruzzaman et al., 2014) and the Phycodnaviridae ranging from 160 to $2550 \mathrm{~kb}$ (Lee et al., 1995; Philippe et al., 2013; Yutin and Koonin, 2013). Viral genome gigantism challenges the traditional definition of viruses conceived as small and simple organisms. Understanding the evolutionary forces acting on the Giant Virus (GV) genomes have profound implication for our knowledge of the viral world and the interplays between cellular organisms and viruses. Additionally, there are accumulating evidences that several 
GVs caused respiratory tract infections. Although the exact mechanism of their pathogenocity is currently unknown, mimiviruses are suspected to be the ethiologic agents of numerous cases of pneumonia acquired by patients in intensivecare institution but also by apparent healthy patients (Kutikhin et al., 2014). Origin of genome gigantism in these families is still a matter of an intense controversy between the advocates of the "genome degradation hypothesis" (Claverie, 2006) and those defending the "genome expansion hypothesis" (Moreira and Lopez-Garcia, 2005; Filee et al., 2008; Yutin et al., 2014). The "genome degradation hypothesis" postulates that Giant Viruses (GVs) derive from a cellular ancestor by progressive genome simplification linked to the adaptation to a parasitic lifestyle. Notably, presence of typical cellular hallmark genes as translational genes, supports the hypothesis that GVs derive from a cellular ancestor (Arslan et al., 2011). However, phylogenetic studies indicate that most, if not all, of these translational genes result from lateral gene transfers (LGTs) from cellular organisms (Moreira and Lopez-Garcia, 2005; Yutin et al., 2014). Indeed, many studies have pointed out the central role of lateral gene transfers during the evolution of GVs (Iyer et al., 2006; Filee et al., 2007, 2008; Moreira and Brochier-Armanet, 2008; Yutin et al., 2013). Finally, gene and genome duplications (Suhre, 2005; Filee and Chandler, 2008) in addition to dissemination of various mobile genetic elements as introns or transposons (Filee et al., 2007; Desnues et al., 2012) have also been identified as important player for GV genome evolution. The combination of these forces support the "genome expansion hypothesis" in which GVs evolved from a relatively simple viral ancestor by progressive gene accretion and duplication. The nature of this ancestor remains speculative but recent discoveries indicate that GVs may derive from DNA transposons belonging to the Polinton/Virophage superfamily (Krupovic and Koonin, 2015). However, experimental data have shown that under laboratory conditions, members of the Mimiviridae can experience rapid genome expansion/contraction. Indeed, under peculiar selective constraints, Poxvirus genomes undergo successive steps of gene duplications and gene losses (Elde et al., 2012). Symmetrically, when mimiviruses are cultivated in bacteria-free media (the preys of their amoebal hosts), numerous genome reductions occur, mainly caused by large deletions (Boyer et al., 2011). On the basis of these data, I recently proposed a model in which GVs evolved using a complex process of "genomic accordion" instead of a general tendency toward either genome expansion or reduction (Filee, 2013). According to this hypothesis, GVs should undergo successive cycles of genome expansion and reduction in order to adapt to modified environmental conditions or new hosts. This hypothesis is readily testable by genomic comparisons of closely related GVs. Thus, I took advantage of the availability of clusters of very similar GVs genomes belonging to the Phycodnaviridae and the Mimiviridae families to analyze the pattern of gene loss/gene gain, using whole genome alignments. In this paper, I show that genomic mutations occur regularly over the time (clock-like). However, a global trend of genome evolution was not observed, each lineage having a specific mode of evolution as duplication and subsequent loss of genes belonging to large multigene families (mimiviruses and megaviruses), recursive insertion and excision of transposons and mobile endonucleases (Chlorella viruses) and acquisition of new genes via LGTs (Ostreococcus and Micromonas viruses). Thus, these observations support the hypothesis that ancestors of the present-day GVs were already GVs that have evolved through a balanced, predominantly neutral process of gene gains and gene losses.

\section{Material and Methods}

\section{Data Collection}

GV genomes were downloaded from the NCBI Viral Genome database at http://www.ncbi.nlm.nih.gov/genome/viruses/. Minimal requirements needed are: at least three nearly complete genomes per group with sufficient genome syntenies to identify orthologous genes without ambiguities. As GVs display numerous gene duplications (Suhre, 2005), synteny is a determinant criterion to recognize the orthologous genes by positional homology. Identical or nearly identical genomes as the three Phaeocystis viruses for example were excluded. The final dataset includes 4 mimiviruses and 4 megaviruses for the Mimiviridae family and 3 Micromonas viruses, 5 Ostreococcus viruses, and 5 Chlorella viruses for the Phycodnaviridae family (Table 1).

\section{Whole Genome Alignment}

In order to identify positional homologs and infer the ancestral state of each gene, multiple genome alignments were carried out and visualized using progressiveMauve (Darling et al., 2004). Whole genome phylogenetic trees based on gene content/substitution distances were computed. Roots of trees were chosen to minimize the evolutionary distance: i.g. the mimivirus tree was rooted with a megavirus and vice versa. Concerning the Chlorella viruses, as the Ostreococcus and the Micromonas virus genomes are too divergent to find reliable genome syntenies, the sequence of Chlorella virus PBCV-1 genome was used. Two by two, whole genome comparisons were achieved with wgVISTA (Couronne et al., 2003) and unaligned DNA segments larger than $300 \mathrm{bp}$ containing at least one ORF were extracted. These sequences were searched with a TBLASTN (Altschul et al., 1990) against a NR database to identify the genomic variations (gene transfers, gene duplications, gene losses, movement of mobile genetic elements, orphan etc...). In order to identify the nature of the genomic events (a gene duplication rather than a gene loss for example), the most parsimonious scenario principle was applied using the whole genome trees as templates. When two scenario are equally parsimonious (implying the same number of mutation steps) the corresponding genes were marked as "ambiguous."

\section{Sequence Analysis and Phylogenies}

Four taxonomic markers (DNA polymerase, A2L Transcripition factor, D5 Primase-helicase, packaging ATPase) were used to build a global phylogenetic tree of the GVs used in this study. The ancestral sequence of each group was reconstructed using FastML (Ashkenazy et al., 2012) and pairwise comparisons were computed using BLASTN (Altschul et al., 1990). 
TABLE 1 | Major characteritics of GV genomes and description of the genomic events that have occured after the divergence with their respective common ancestors.

\begin{tabular}{|c|c|c|c|c|c|c|c|c|c|c|c|c|c|}
\hline $\begin{array}{l}\text { Virus } \\
\text { name }\end{array}$ & Genbank & $\begin{array}{l}\% \text { Identity } \\
\text { ancestor }\end{array}$ & $\begin{array}{l}\text { Size } \\
\text { (kb) }\end{array}$ & Duplication & LGT & Insertion & $\begin{array}{l}\text { Total } \\
\text { Gain }\end{array}$ & Loss & Excision & $\begin{array}{l}\text { Total } \\
\text { loss }\end{array}$ & $\begin{array}{l}\text { Unknow } \\
\text { (Orphan) }\end{array}$ & Translocation & $\begin{array}{c}\text { Genomic } \\
\text { Events }\end{array}$ \\
\hline Mimivirus & NC_014649.1 & 99 & 1182 & 5 & 0 & 1 & 6 & 7 & 0 & 7 & 0 & 0 & 13 \\
\hline Mamavirus & JF801956.1 & 99 & 1192 & 5 & 0 & 0 & 5 & 7 & 0 & 7 & 3 & 8 & 23 \\
\hline Hirudovirus & KF493731 & 99 & 1181 & 3 & 0 & 1 & 4 & 6 & 0 & 6 & 0 & 1 & 11 \\
\hline Terra2 & NC_023639 & 99 & 1169 & 10 & 3 & 2 & 15 & 19 & 1 & 20 & 3 & 3 & 41 \\
\hline Megavirus Chiliensis & NC_016072.1 & 98 & 1259 & 9 & 1 & 2 & 11 & 5 & 0 & 5 & $4(0)$ & 0 & 20 \\
\hline Megavirus lba & NC_020232 & 97 & 1231 & 9 & 0 & 1 & 10 & 24 & 0 & 24 & $4(0)$ & 1 & 39 \\
\hline Megavirus courdo11 & JX975216 & 98 & 1246 & 6 & 1 & 3 & 10 & 15 & 1 & 16 & $10(2)$ & 0 & 36 \\
\hline Megavirus terra1 & NC_023640 & 99 & 1245 & 12 & 0 & 1 & 13 & 8 & 1 & 9 & $1(0)$ & 0 & 23 \\
\hline OtV1 & NC_013288 & 88 & 192 & 3 & 5 & 0 & 8 & 9 & 0 & 9 & $4(1)$ & 0 & 21 \\
\hline OtV2 & NC_014789.1 & 87 & 184 & 1 & 13 & 0 & 14 & 12 & 0 & 12 & $7(4)$ & 0 & 33 \\
\hline Osv5 & NC_010191 & 89 & 185 & 1 & 5 & 0 & 6 & 4 & 0 & 4 & $7(4)$ & 0 & 17 \\
\hline OIV1 & NC_014766 & 85 & 194 & 1 & 11 & 0 & 12 & 8 & 0 & 8 & $4(2)$ & 0 & 24 \\
\hline OtVRT-2011 & JN225873 & 92 & 190 & 0 & 12 & 0 & 12 & 7 & 0 & 7 & $18(9)$ & 0 & 37 \\
\hline MpV SP1 & JF974320 & 82 & 173 & 1 & 8 & 0 & 9 & 20 & 0 & 20 & $14(3)$ & 0 & 43 \\
\hline MpV1 & NC_014767 & 83 & 184 & 0 & 8 & 0 & 8 & 16 & 0 & 16 & $27(16)$ & 0 & 51 \\
\hline MpV PL1 & HQ633072 & 81 & 197 & 12 & 9 & 0 & 21 & 17 & 0 & 17 & 23(15) & 0 & 61 \\
\hline Nys-1 & JX997183 & 98 & 348 & 3 & 0 & 3 & 6 & 13 & 2 & 15 & $6(1)$ & 0 & 27 \\
\hline NY2A & NC_009898 & 94 & 369 & 16 & 4 & 6 & 26 & 14 & 1 & 15 & $7(5)$ & 0 & 48 \\
\hline AR158 & NC_009899 & 99 & 345 & 7 & 3 & 2 & 12 & 9 & 4 & 13 & $2(1)$ & 0 & 27 \\
\hline IL-5-2s1 & JX997170 & 99 & 345 & 1 & 0 & 1 & 2 & 3 & 3 & 6 & $3(0)$ & 0 & 11 \\
\hline \multirow[t]{2}{*}{ MA-1D } & JX997172 & 99 & 340 & 0 & 0 & 2 & 2 & 9 & 6 & 15 & $2(1)$ & 0 & 20 \\
\hline & & & & 105 & 83 & 25 & 212 & 232 & 19 & 251 & $150(74)$ & 13 & 626 \\
\hline
\end{tabular}

Sequences were aligned using MUSCLE (Edgar, 2004) with default parameters and conserved parts of the alignments usable for phylogenetic analyses were chosen using Gblocks (Castresana, 2000). Concerning phylogenies, best-fitting ML models were selected using Protest (Abascal et al., 2005) and the trees were implemented using PhyML 3.0 (Guindon et al., 2009). Branch supports were calculated using a LRT ShimodairaHasegawa (SH) procedure.

Raw data including whole genome alignments, gene sequence alignments, reconstructed ancestral sequences, phylogenetic trees etc. are available at http://echange.legs.cnrs-gif.fr:5000/ fbsharing/b95jcnuZ.

\section{Results}

\section{A Predominant Model of Neutral Evolution due to Regularly Accumulated Mutations over the Time}

The pattern of gene loss/gene gain at a small evolutionary scale for five groups of closely related GVs belonging to the families Megavirales and Phycodnaviridae was analyzed. Up to 626 genomic events were detected (Table 1) corresponding to 212 gene gains, 251 gene losses, and 163 ambiguous variants (including 74 orphans). Insertion and excision of mobile genetic elements are additional factors (44) and very few gene translocations have been evidenced (13). Gene duplication (105 events), lateral gene transfers (85) and gene losses (232) were by far the major evolutionary forces shaping the GV genomes.

For each group of GVs, ancestral sequence reconstruction of four taxonomic gene markers was computed. Percentages of identity between these ancestral sequences and the present ones were indicated in Table 1. If a molecular clock of sequence evolution is assumed, this analysis provides inside each group of viruses, a proxy of the time of divergence between each viruses and the last common ancestor of their respective groups. Thus, mimiviruses, megaviruses, and Chlorella viruses appear closely related (94 to $99 \%$ of sequence identity compared to their respective ancestral sequences) whereas Ostreococcus and Micromonas viruses are substantially more divergent (81 to $92 \%$ of sequence identity). Figure 1 plots the number of genomic events per $\mathrm{kb}$ for each virus, with respect to the sequence identity of the four marker genes. The regression line indicated that there is a negative correlation between the quantity of genomic variations and the sequence identity. In other words, the amount of genomic variations increased linearly with the time of divergence from the last common ancestor. There is no evident deviation from the regression line indicating slow down or increase of the rate of genomic mutation for peculiar groups or individual virus. Consequently, these data support the idea that GV genomic variations accumulate regularly over the time in an approximately clock-like manner. Adaptive genomic mutations 


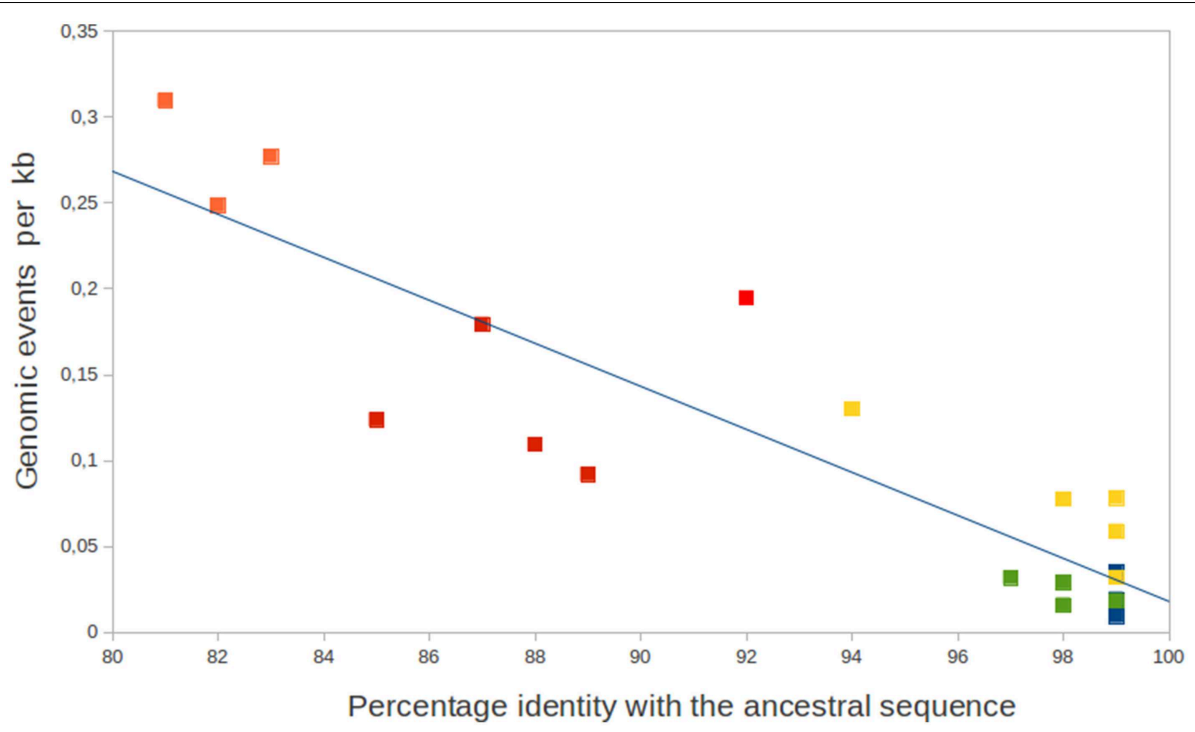

FIGURE 1 | Number of genomic events per kb for each GVs with respect to the percentage of nucleotide similarities of the four taxonomic genes with the ancestral sequences. GVs are represented by squares: orange for Micromonas viruses, red for Ostreococcus viruses, green for Chlorella viruses, yellow for megaviruses, and blue for mimiviruses. The blue line represents the regression $\left(r^{2}=0.81\right)$. could occur at a constant rate but a predominant neutral model of genomic evolution seems more possible.

\section{Evidences for Group-specific Evolutionary Patterns}

Analyses of the spatial patterns of gene loss and gain along the genomes were displayed in Figure 2 (mimiviruses and megaviruses) and Figure 3 (Ostreococcus and Chlorella viruses). With the exception of the megavirus LBA, most of mimiviruses and megaviruses display an equilibrium between gains and losses. The megavirus LBA has significant higher number of gene losses than gene gains leading to a smaller genome compared to the other megaviruses (Table 1). As previously observed, these results confirm that the extremities of the Mimiviridae genomes are hot spots of genome variation (Filee et al., 2007; Colson et al., 2011). In addition, most of the genomic variations in the two viral groups concern multigenic families: more than $75 \%$ of duplications concern families with at least three additional representatives in the corresponding genomes and $50 \%$ of the gene losses also impact duplicated families. Duplicated genes families represent roughly one third of the total genes content of the mimivirus (Suhre, 2005) and of the megavirus chiliensis (Arslan et al., 2011). This over-representation of duplicated families compared to their current distribution in the Mimiviridae genomes suggests that they are the primary source of variation in this viral group. Indeed, LGTs, movement of genes or mobile genetic elements as transposon or mobile endonuclease and generation of orphans represent an anecdotal fraction of the genomic variation identified in this study. The rarity of LGTs is somewhat surprising, as many studies have pointed out their importance during the evolution of the Mimiviridae (Iyer et al., 2006; Filee et al., 2007, 2008; Moreira and Brochier-Armanet, 2008; Yutin et al., 2013).
The Phycodnaviridae family displays clearly different patterns of genome evolution (Figure 3). Ostreococcus viruses demonstrate a slight tendency of genome expansion for most of their representatives whereas Chlorella viruses display very different orientations including significant genome reduction for MA-1D and NYs-1, significant genome expansion for NY2A and relative stability for the others. A similar situation is observed for the three Micromonas virus genomes (Table 1). Another striking difference between the Phycodnaviridae and the Mimiviridae is the location of the genomic variations: there are scattered along the genomes for the Phycodnaviridae rather than being concentrated at the extremities as observed for the Mimiviridae. Interestingly, a study of laboratory variants of Chlorella viruses has evidenced the occurrence of large deletions at the left ends of the genomes (Landstein et al., 1995). However, an analysis of naturally occurring Chlorella viruses showed that insertion/deletion are more disseminated, corroborating our results (Nishida et al., 1999). Insertion and excision of mobile genetic elements represent a significant part of the Chlorella virus genomic mutations (23\% of the total events). This observation confirms the importance of the GIY-YIG mobile endonuclease, associated or not with introns, and IS607 transposons during the genome evolution of the Chlorella viruses (Filee et al., 2007). Chlorella viruses also display an important number of multigene families gene losses/gene duplications. which account for $40 \%$ of the genomic events whereas they represent only $15-20 \%$ of the total gene content (Filee et al., 2008). Conversely, Ostreococcus virus and Micromonas viruses do not display any mobile elements movement and have a low amount of gene duplication (Figure 1, Table 1). Another difference is the importance of LGTs in Ostreococcus and Micromonas viruses. LGT is by far the major evolutionary force acting on the genomes of these viruses representing more than one third of the total number of 


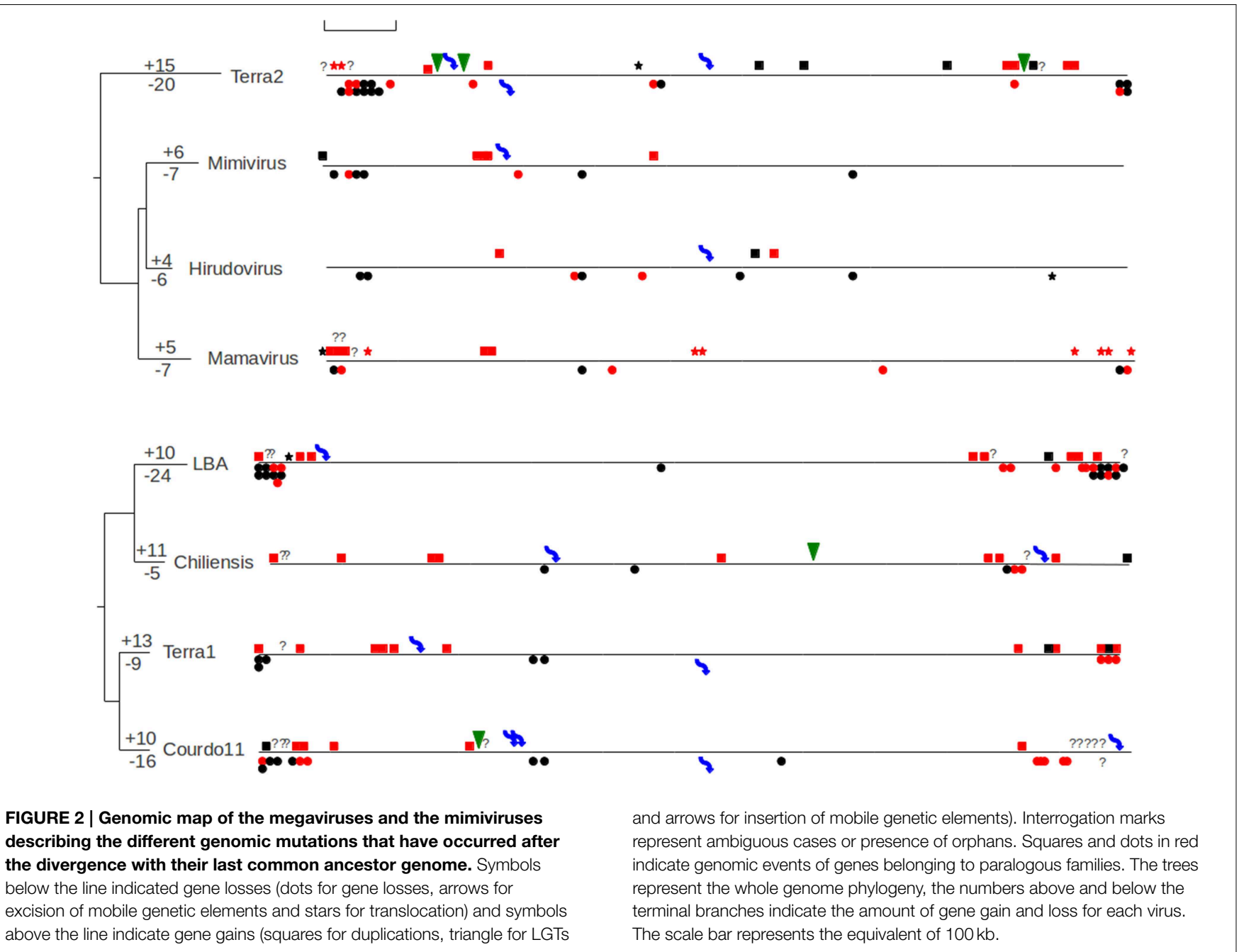

mutations observed here. This observation contrasts with the rarity of LGT observed in the other GVs.

Taken together, these data suggest that there is no general trend of genome expansion or reduction in the family. Indeed, each group of viruses displays specific patterns of gene gain/gene loss. mimiviruses, megaviruses, and Chlorella viruses are mainly affected by gene loss and duplication targeting the multigene families. Multigene families appear as the main evolutionary tools in these viruses. Ostreococcus and Micromonas viruses are principally affected by LGTs and display few events of gene duplications.

\section{Characterization of the LGTs Involved a Large Diversity of Sources and Protein Functions}

Genomic comparison of closely related GVs lead to the identification of 71 individual cases of gene transfers (Supplementary Table 1). Representative sets of phylogeny of these genes with identifiable functions are given Figure 4. As previously noticed, the vast majority of the LGTs concern the Ostreococcus and Micromonas viruses. Indeed, only 4 transfers for the Chlorella Phycodnaviruses and 5 for the mimiviruses and the megaviruses were observed. Most of these transfers concern genes coding proteins of unknown functions. Among the 24 genes displaying strong sequence similarity with genes associated with identifiable functions, 9 are homologs of DNA methyl transferase/methylase genes. These enzymes are components of Restriction-Modification systems, a prokaryotic system of defense against invading DNA. These enzymes are prone to LGTs and may behave as selfish mobile genetic elements (Kobayashi, 2001). The other functions implicated in LGTs compose a heterogeneous set of diverse informational or metabolic proteins: a Cytochrome B5, a Heat Shock protein, a RNA polymerase sigma factor etc. Interpretation of the phylogenies is generally unequivocal: GV genes are most often well nested among a forest of bacterial or eukaryotic genes (MpV-201, MpVPL1_135, OtV2_29, OtV2_30, NY2A_137, OtV2_78 + OlV1_89, OtVRT-2011_115) (Figure 4A). Thus, this situation strongly favors a LGT polarized from the cell to the virus. Sometimes, the GVs sequences appear as a sister group of the host sequences, well nested inside the eukaryotic sub-tree (OtV2_200/OlV1_214, OtV2_222/OlV1_234, OtVRT-2011_115 etc...) (Figure 4B). This pattern indicates strongly that the 


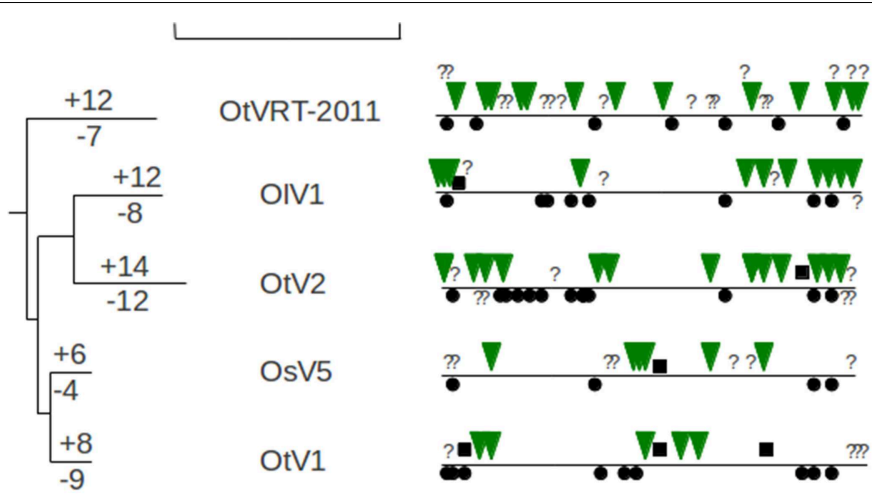

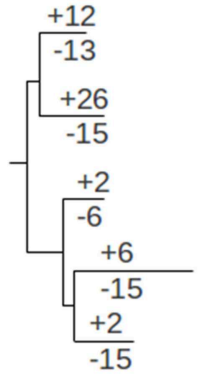

FIGURE 3 | Genomic map of the Chlorella and Ostreococcus Phycodnaviruses describing the different genomic mutations that have occurred after the divergence with their last common ancestor. Symbols below the line indicated gene losses (dots for gene losses, arrows for excision of mobile genetic elements) and symbols above the line indicate gene gains (squares for duplications, triangle for LGTs and arrows for insertion of mobile genetic elements). Interrogation marks represent ambiguous cases or presence of orphans. Squares and dots in red indicate genomic events of genes belonging to paralogous families. The trees represent the whole genome phylogeny, the numbers above and below the terminal branches indicate the amount of gene gain and loss for each virus. The scale bar represents the equivalent of $100 \mathrm{~kb}$. viruses have acquired the genes from their algal hosts. However, more ambiguous patterns are also encountered (Figure 4C): in the OtVRT-2011_237 tree, the Ostreococcus viral sequence is located close to host sequence but distantly related from the other eukaryotic sequences (the tree has been rooted with Bacterial sequences). In this case, the polarization of the transfers is unclear. However, the fact that this gene is absent in all Phycodnaviruses, with the exception of OtVRT-2011, favors the hypothesis of a gene transfer from the cells to the virus. The NY2A_359/NY2A_543/AR158_487 gene phylogeny is even more puzzling: several Phycodnaviridae and Mimiviridae sequences are represented but form a deeply polyphyletic group. In this case, a possible explanation involves two independent LGTs from bacteria. Alternatively the sequence NY2A_359 has been acquired from another GVs, possibly a Mimiviridae infecting "Alga" as a Phaeocystis virus or an Aureococcus virus. Finally, in the MpV1_42 gene phylogeny, the viral sequence appears as a sister taxa with the Ostreococcus lucimarinus sequence (a potential host). However, the GV and host sequences are also closely related with diverse cyanobacterial sequences and their associated phages. This situation leads to two possible scenarios:

- The "two steps scenario" implies first, acquisition of the bacterial gene by MpV1 and second, transfers of the viral genes to the host genome.
- Alternatively, it could be envisaged independent captures of the bacterial gene by the virus and the alga.

The "two steps scenario" could provide an interesting situation in which the virus has served as a "shuttle" for an inter-kingdom LGT.

Prokaryotes represent the predominant source of genes acquired by LGTs (Figure 5). Various phyla are involved including Cyanobacteria, Proteobacteria, Firmicutes, Spirochetes, Verrucomicrobia... (Supplementary Table 1). Genes derived from the hosts and from other eukaryotic organisms represent additional but minor sources. These include mainly fungi and various marine organisms (Hydra, the anemone Nematostella, the crustacean Daphnia, the ascidian Ciona, diverse alga as Chlorella or Cyanidioschyzon etc...). Finally, 4 virus-to-virus LGTs were also identified, two implicating other GVs (a Marseillevirus and a Mimivirus), the two others ones with Cyanophages. Moreover, a substantial fraction of the LGTs displays ambiguous phylogenies blurring the identification of the donors (Figure 5). Possible additional cases of virus-to-virus LGTs are also possible (OtV2_40, OtVRT-2011_55, MpV1_243, MpVPL147...). As discussed previously with the NY2A_359/NY2A_543/AR158_487 gene phylogeny, the trees display complex evolutionary histories with possible multiple and independent acquisitions of the 


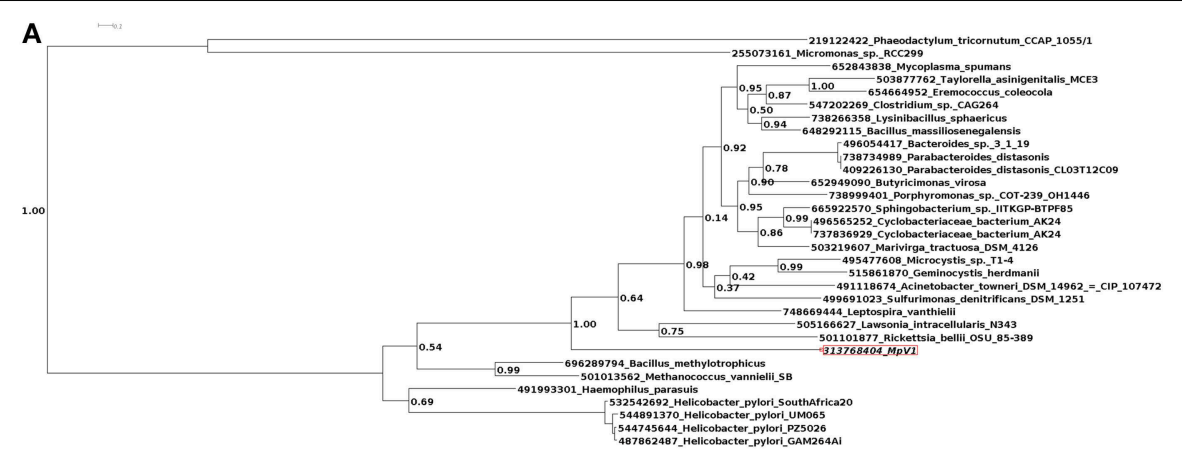

MpV1_201 /cytosine-C5 specific DNA methylase

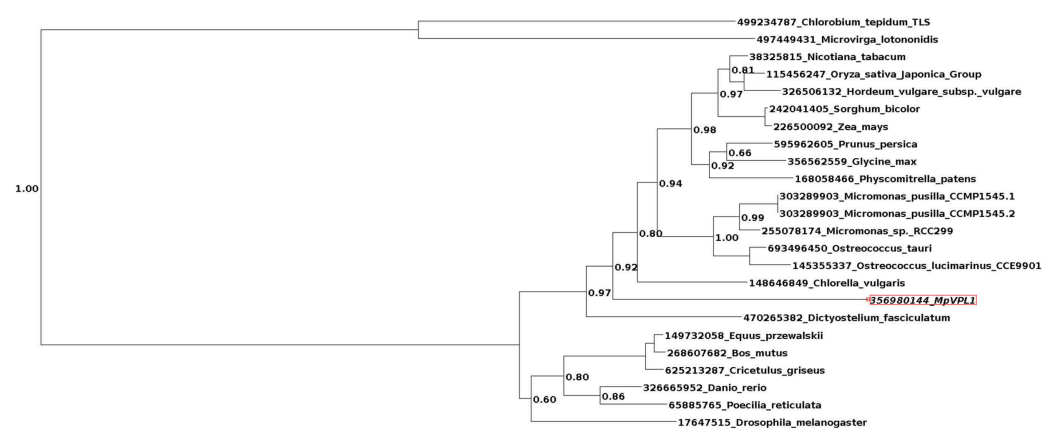

MpVPL1_135 / heat shock protein

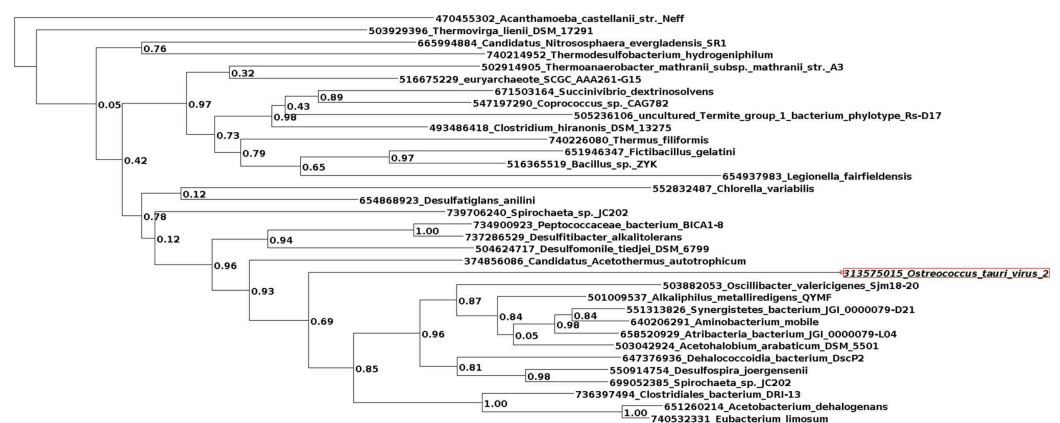

OtV2_29 / 3-methyl-2-oxobutanoate hydroxymethyltransferase

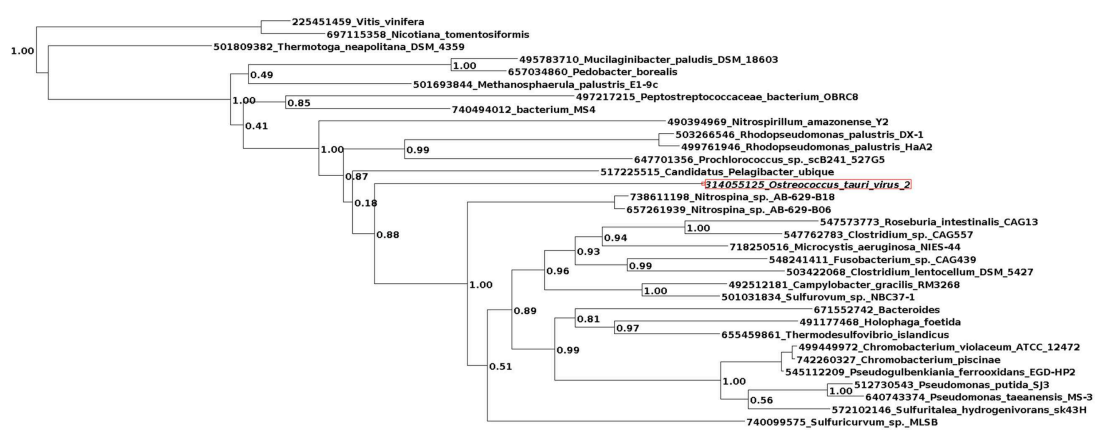

OtV2_30 / acetolactate synthase 


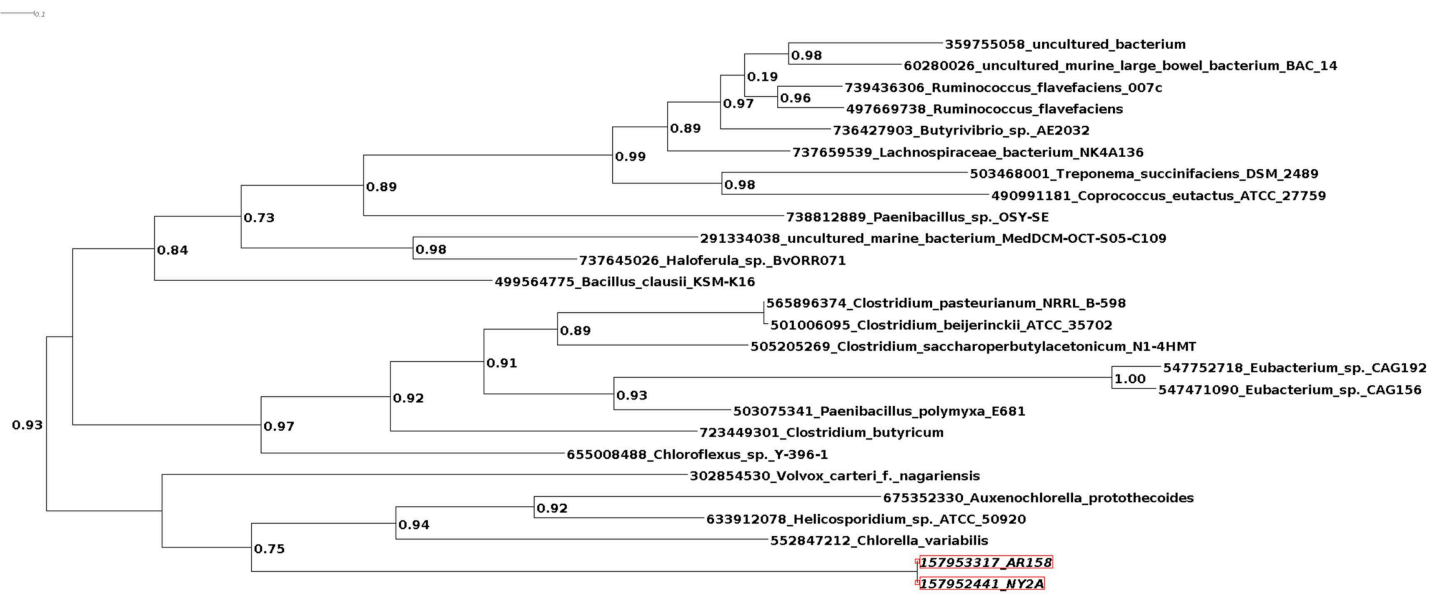

Ny2A_137+AR158_126 / Glucan endo-1,3-beta-glucosidase

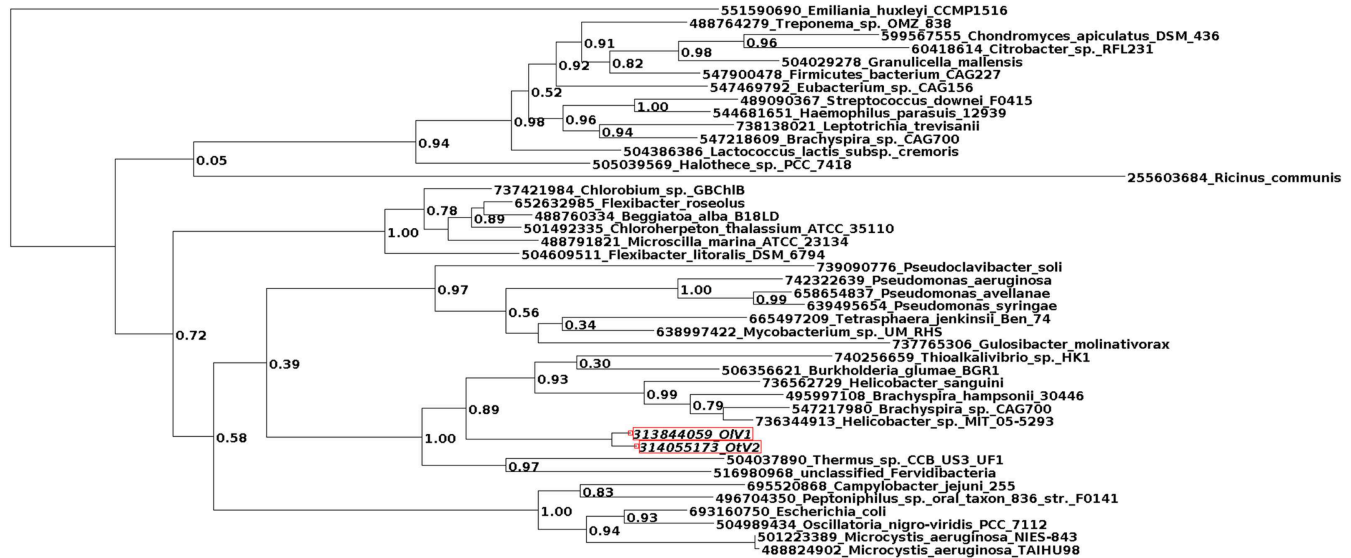

OtV2_78 + OlV1_89 / acetolactate synthase

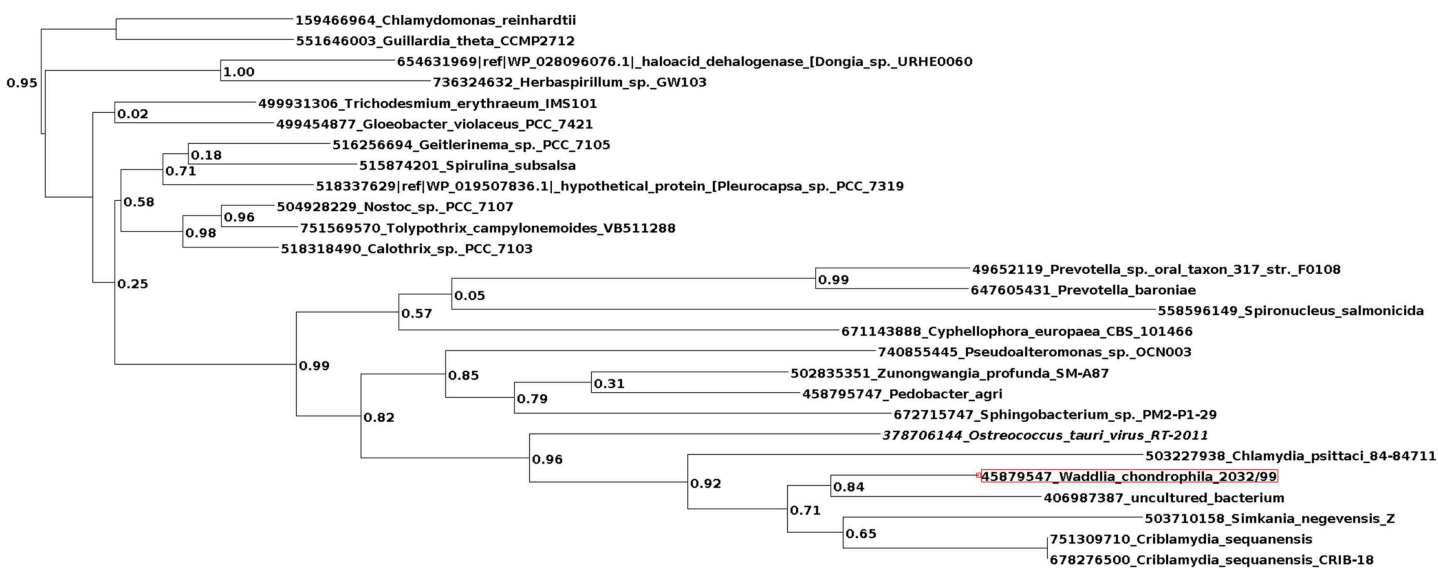

OtVRT-2011_37 / HAD-superfamily hydrolase 


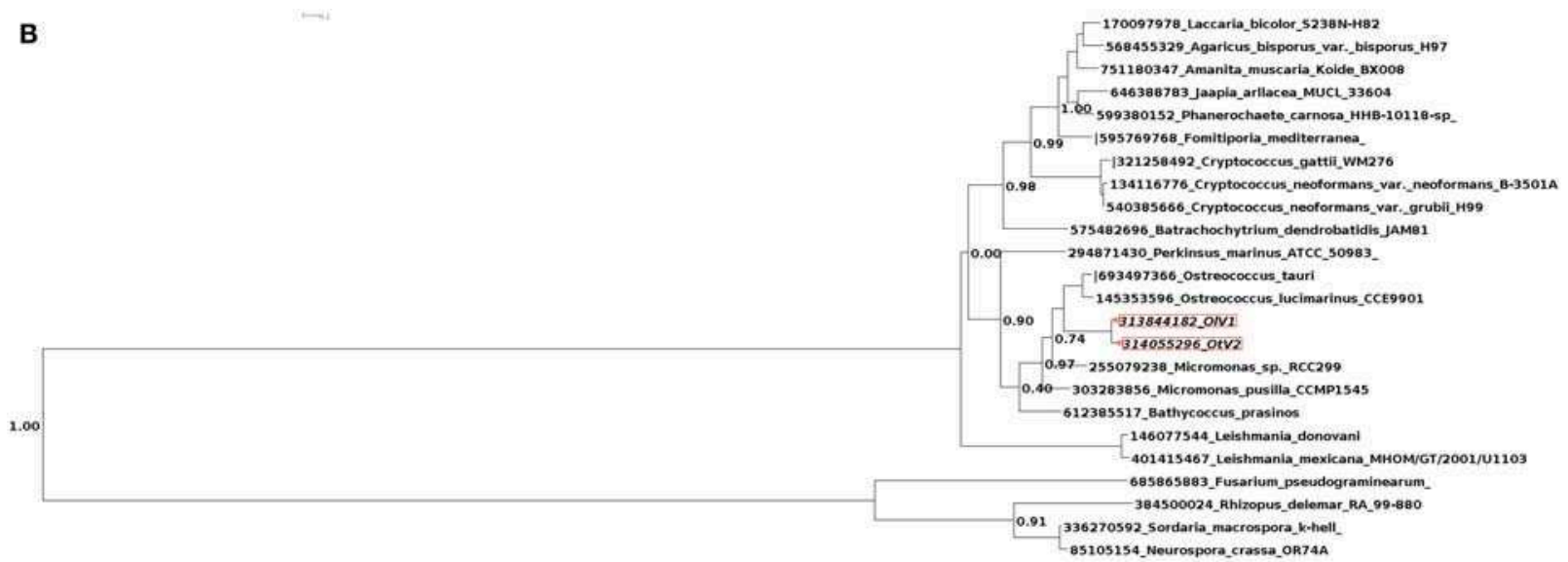

\section{OtV2_200 + OIV1_214 / RNA-polymerase sigma factor}

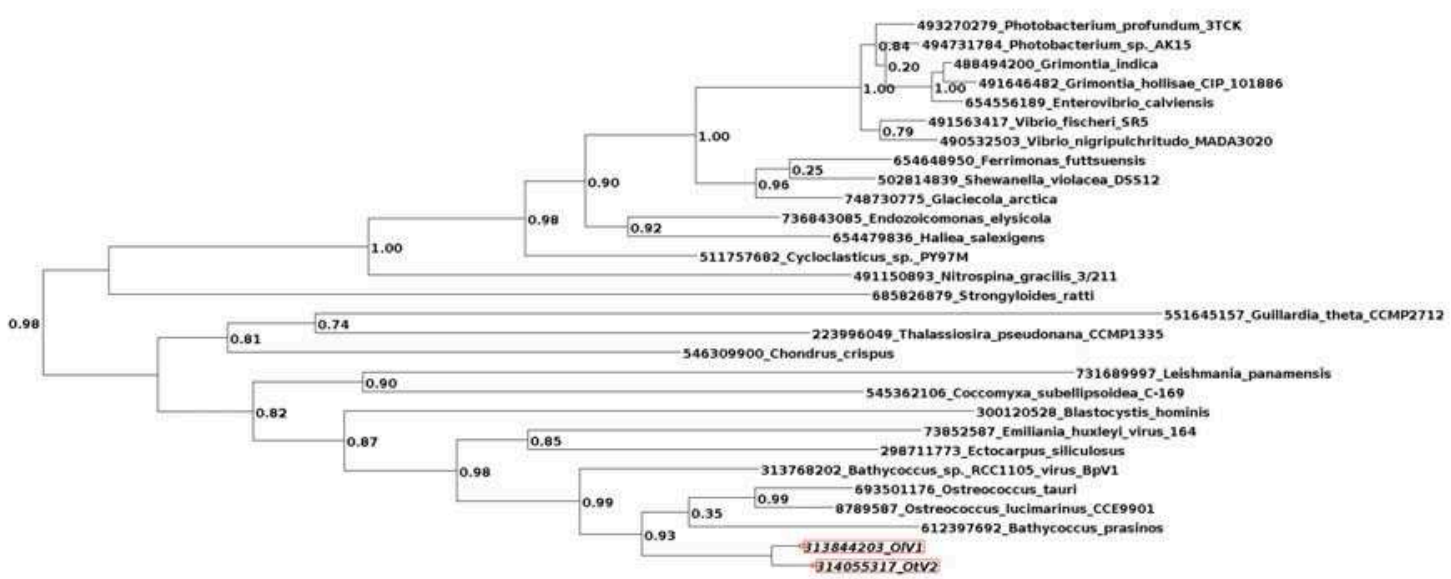

OtV2_222 + OlV1_234 / PiT family transporter

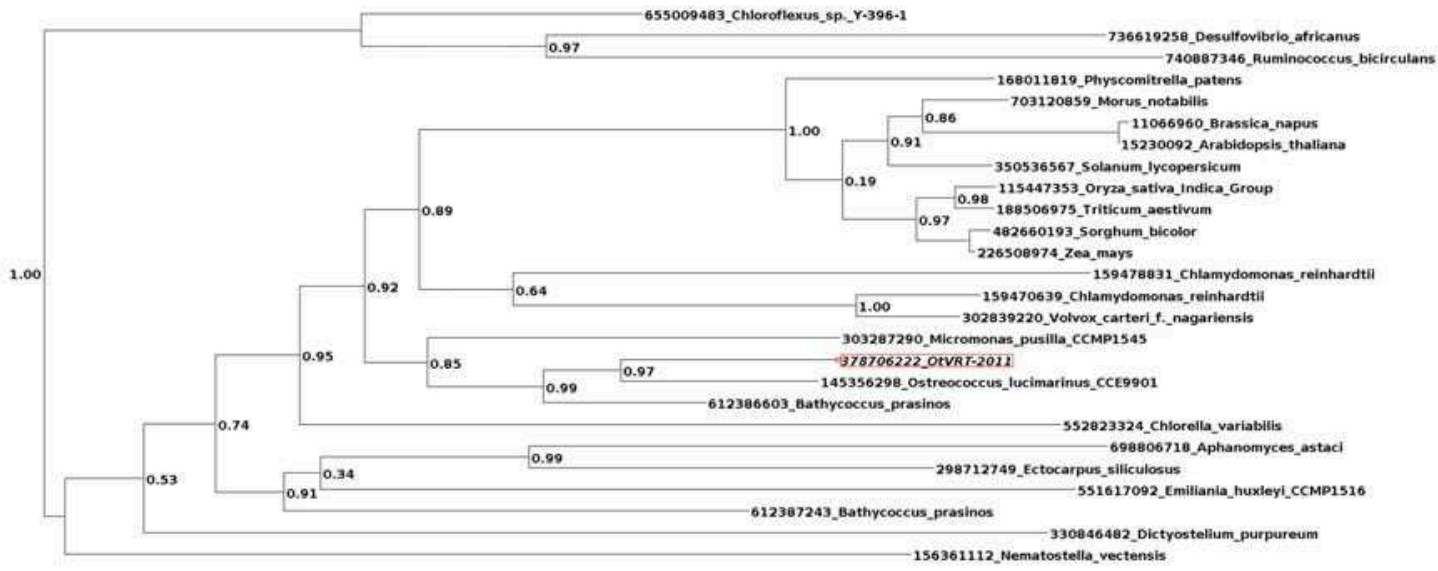

OtVRT-2011_115 / Ammonium transporter 


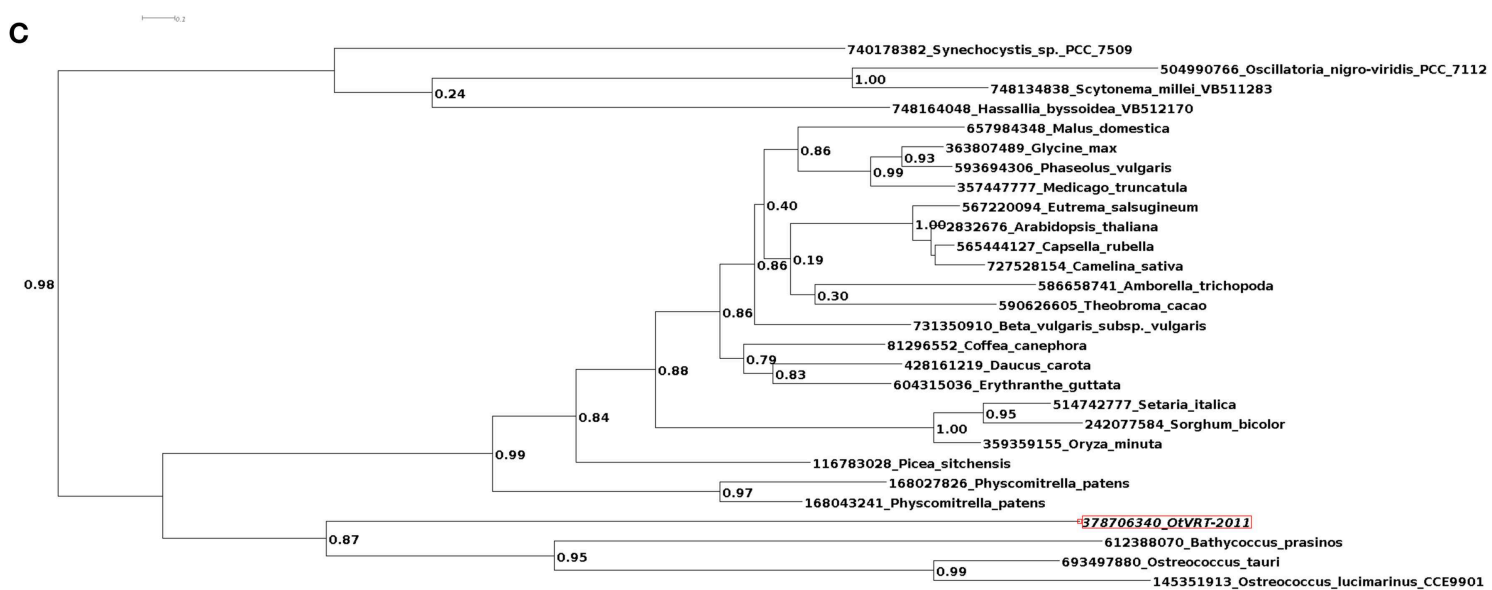

\section{OtVRT-2011_237 / ubiquinol oxidase 4}

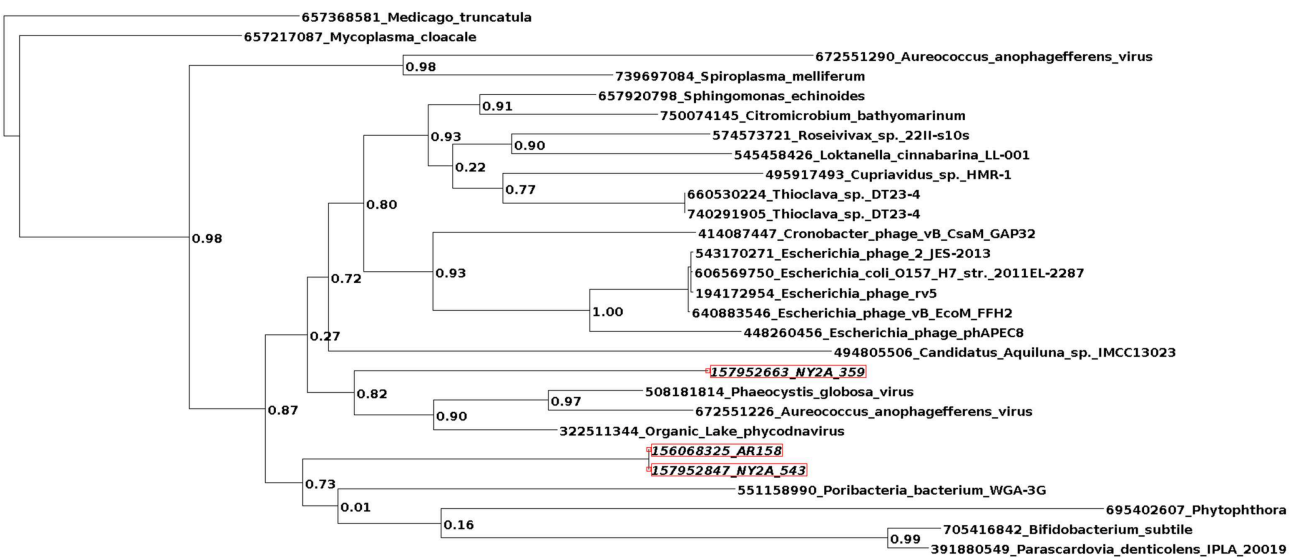

Ny2A_359 + Ny2A_543+AR158_487 / DNA adenine methyltransferase

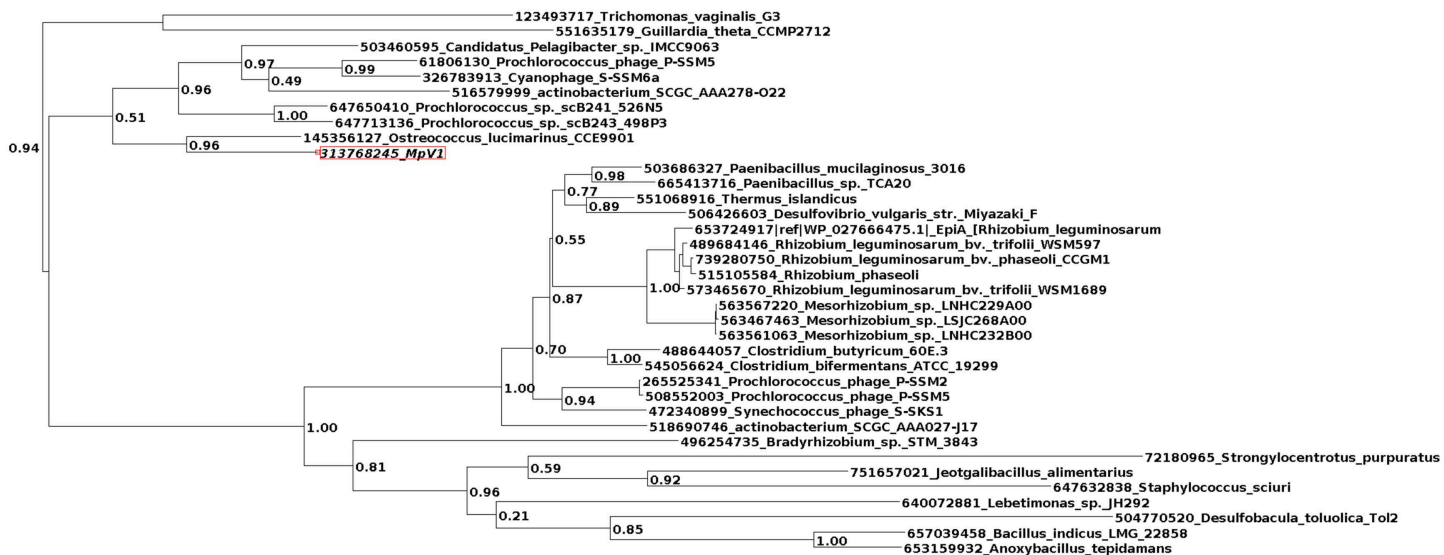

\section{MpV1_42 / UDP-glucose 4-epimerase}

FIGURE 4 | Phylogenies of different cases of LGT encountered in GVs. (A) LGT from diverse Bacteria and Eukarya (B) LGT from the hosts. (C) LGT from ambiguous sources. Viral sequences have been framed in red. The numbers beside each node indicate the value of the SH-like statistical test. 


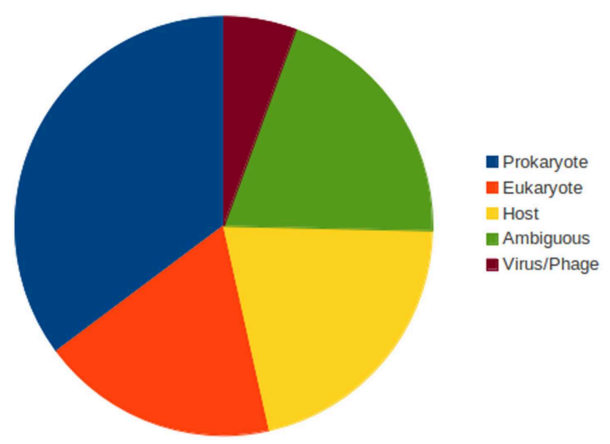

FIGURE 5 | Taxonomic sources of the LGTs.

genes by the viruses rather than LGTs between the viruses themselves.

In summary, more than 70 cases of LGTs with a clear polarization from cells to the GVs have been evidenced. These transfers concern genes encoding proteins of very diverse functions and prokaryotes are the predominant source of gene acquisitions. LGTs from the hosts and from other eukaryotic organisms also occurred and isolated cases of gene transfers between viruses, including GV-to-GV LGTs, are also reported.

\section{Discussion}

Comparative genome analysis of closely related GVs revealed that there are no general trends of genome reduction or genome expansion. Genomic mutations occur at a clock-like, constant rate, in both GV lineages but each group displays specific patterns of genome evolution:

- GVs with the largest genome as the Mimiviridae (mimiviruses and megaviruses) evolved mainly by a balanced process of duplications and losses of multigene families. Paralogous gene families appear as a genomic toolbox by providing most of the new genes but subsequent losses equilibrate the game and genome expansion is not discernible. LGTs and movements of mobile genetic elements are additional but marginal forces of genome evolution.

- Viruses with the smallest genomes as the Ostreococcus and Micromonas phycodnaviruses derive most of their genetic novelties by LGTs from a large variety of sources including various prokaryotic and eukaryotic species. Again, gene losses have balanced the process and globally the genomes appear with a relatively stable size.

- Chlorella phycodnaviruses which have intermediate genome sizes display more complex patterns of evolution, combining gene duplications and gene losses of multigene families in addition to numerous insertion/excision of IS607 Insertion Sequences and GIY-YIG mobile endonucleases. At the opposite, LGTs are rarely observed. These processes lead one of these Chlorella viruses to significant genome expansion, whereas the others display some genome reduction or genome size stability. It is noteworthy that the data support the fact that these mobile genetic elements are effectively able to transpose in the GV genomes. Their presences do not result from successive and independent LGTs from cellular sources. Abundance of these selfish genetic elements in GV genomes is a very specific feature among eukaryotic viruses (Filee et al., 2007). Indeed with the exception of the polydnaviruses that have a symbiotic, intra-cellular life style (Dupuy et al., 2011), mobile genetic elements are absent or very rare in eukaryotic viruses (Gilbert et al., 2014). Thus, the results presented here indicate that their importance during the evolutionary history of the GVs should not be underestimated.

It should be noted that the Ostreococcus and the Micromonas viruses are more distantly related from each others (80-90\% identity of the four taxonomic markers with the ancestral sequence) than the Mimiviridae (97-99\%) and the Chlorella viruses (94-99\%). Despite their long period of divergence, the genome synteny of the Ostreococcus and the Micromonas viruses are still well conserved. By opposition, the genome colinearity has been largely disrupted between the mimiviruses and the megaviruses: only a $600 \mathrm{~kb}$ central segment is conserved (Arslan et al., 2011; Yoosuf et al., 2012). Gene duplication and gene losses that occur at high rate at the extremities of the Mimiviridae genomes explain the fast disruption of the genome synteny. Similar processes are acting on the Chlorella virus genomes but the repartition of the genomic mutations is not biased toward the extremities. Among the 35 partial or nearly complete Chlorella virus genomes, this process leads to a fast and complete disappearance of the synteny, even for relatively close viruses (Jeanniard et al., 2013). Thus, the remarkable level of genome co-linearity of the Micromonas and Ostreococcus viruses despite a long period of divergence from their common ancestor might reflect fundamental different evolutionary processes compared to the GVs with larger genomes. Additionally, specific constraints on the genome size, for example for the packaging of the DNA into the capsid, might also limit the extent of genome variation and expansion. Interestingly, Mimiviridae and Chlorella Viruses display a large spectra of host specificity compared to Ostreococcus and Micromonas viruses. Mimiviruses have been isolated from Amoeba, but recent data indicate that they are also found in human (Kutikhin et al., 2014), leeche (Boughalmi et al., 2013), or hydra (Grasis et al., 2014). Chlorella phycodnaviruses are able to infect a large variety of protists harboring Chlorella symbiont (Van Etten, 2003) but can also infect mammals (Yolken et al., 2014). By contrast, Ostreococcus viruses display a strong host specificity (Clerissi et al., 2012). Thus, it is tempting to suggest that the broad host range of mimiviruses and Chlorella viruses are linked to their propensity to generate paralogous gene families, allowing the viruses to adapt to new hosts or changing ecological niches. In support to this hypothesis, some of the larger multigene families encode virus-host interaction functions (ankyrin-repeat, protein kinase, glysosyl transferase, FNIP-like repeats etc...). Interestingly this hypothesis offers further developments to determine the genes and molecular mechanisms which allow these GVs to adapt to a wide range of hosts, including diverse mammals, including human in relation with their potential health care concerns. 
The paucity of LGTs detected in the genome of the Mimiviridae and the Chlorella viruses examined here is intriguing as many studies pointed out the importance of gene transfers during the evolution of these groups (Iyer et al., 2006; Filee et al., 2007, 2008; Moreira and Brochier-Armanet, 2008; Yutin et al., 2013). It was proposed that the sympatric lifestyle of these viruses with various prokaryotic species (symbionts or preys of their protist hosts) have facilitated the access to a very diverse gene pool as sources of new genes and functions (Filee et al., 2007; Raoult and Boyer, 2010). However, we identified only 4 LGTs for the Chlorella viruses and 5 for the Mimiviridae. This observation raises the question of the overestimation of the importance of LGTs during the evolution of the GVs. An important point is the determination of the polarization of the transfers and the interpretation of the phylogenies. A large fraction of phylogenies including viral and cellular genes are inconclusive: low level of resolution, complex patterns involving multiple transfers/losses, ambiguities in the polarization as previously stated (Forterre, 2010). Interestingly, acquisition of numerous GV genes by various eukaryotes has been evidenced recently (Filee, 2014; Maumus et al., 2014). Thus, some LGTs wrongly polarized from the cell to the virus correspond in turn to acquisition of GV genes by cells. However, an important fraction of the GV genes possibly acquired from cells have homologs only in prokaryotes (Filee et al., 2007). In this case, the polarization of the transfers is unequivocal as it seems unlikely that prokaryotes frequently acquired genes from eukaryote viruses. Interestingly, the major part of LGTs identified in this study concern genes from bacterial origin suggesting that the possible over-estimation of the importance of LGTs essentially concerns genes from eukaryotic (host) origins. Finally, LGTs are the most frequent genomic events observed in the Ostreococcus and Micromonas viruses, but these viruses are distantly related to each other compared to the Mimiviridae and Chlorella viruses. This suggests that LGTs play a significant role in GV evolution, but they probably arise at a lower rate than previous thought for several GV lineages in which gene duplications/losses or movements of mobile genetic elements are the prevalent evolutionary forces. Additionally, it is also

\section{References}

Abascal, F., Zardoya, R., and Posada, D. (2005). ProtTest: selection of best-fit models of protein evolution. Bioinformatics 21, 2104-2105. doi: 10.1093/bioinformatics/bti263

Altschul, S. F., Gish, W., Miller, W., Myers, E. W., and Lipman, D. J. (1990). Basic local alignment search tool. J. Mol. Biol. 215, 403-410. doi: 10.1016/S00222836(05)80360-2

Arslan, D., Legendre, M., Seltzer, V., Abergel, C., and Claverie, J. M. (2011). Distant Mimivirus relative with a larger genome highlights the fundamental features of Megaviridae. Proc. Natl. Acad. Sci. U.S.A. 108, 17486-17491. doi: 10.1073/pnas.1110889108

Ashkenazy, H., Penn, O., Doron-Faigenboim, A., Cohen, O., Cannarozzi, G., Zomer, O., et al. (2012). FastML: a web server for probabilistic reconstruction of ancestral sequences. Nucleic Acids Res. 40, W580-W584. doi: 10.1093/nar/gks498

Boughalmi, M., Pagnier, I., Aherfi, S., Colson, P., Raoult, D., and La Scola, B. (2013). First isolation of a giant virus from wild Hirudo medicinalis possible that many LGTs identified previously in the literature have occurred early, before the diversification of the different GV families. Supplementary data with a broader viral genome sampling and comparisons of more distantly GVs are clearly needed to address the exact importance of LGTs in the evolution of this viral family.

Taken together, these interpretations are not compatible with the hypothesis indicating that GVs derive from a cellular ancestor via progressive genome degradation in each lineage (Claverie, 2006). These data also reject the "pick pocket" hypothesis in which GVs experience massive LGTs leading to genome expansion (Moreira and Lopez-Garcia, 2005). By contrast, these data support the idea of a "genomic accordion" in which GVs evolved through a balanced process of gene gains (duplication mainly but also LGTs) compensated by gene losses. Indeed, each GV lineage most likely has a specific pattern of genome evolution, probably reflecting different host-virus interactions. However, there is little doubt that the last common ancestor of the Megavirales was a relatively simple virus, encoding a limited genomic repertoire that has successively expanded over the times (Yutin et al., 2014; Krupovic and Koonin, 2015). Our results indicate that the rate of genome expansion has slow down before the diversification of the Mimiviridae and the Chlorella viruses. Thus, these data support the idea that the ancestor of these viral families was already a GV, indicating that genome gigantism has emerged early during the evolution of the Megavirales (Yutin et al., 2009).

\section{Acknowledgments}

I would like to thanks Jean Michel Rossignol for useful comments and careful reading of the manuscript.

\section{Supplementary Material}

The Supplementary Material for this article can be found online at: http://journal.frontiersin.org/article/10.3389/fmicb. 2015.00593/abstract leech: Mimiviridae isolation in Hirudo medicinalis. Viruses 5, 2920-2930. doi: 10.3390/v5122920

Boyer, M., Azza, S., Barrassi, L., Klose, T., Campocasso, A., Pagnier, I., et al. (2011). Mimivirus shows dramatic genome reduction after intraamoebal culture. Proc. Natl. Acad. Sci. U.S.A. 108, 10296-10301. doi: 10.1073/pnas.11011 8108

Castresana, J. (2000). Selection of conserved blocks from multiple alignments for their use in phylogenetic analysis. Mol. Biol. Evol. 17, 540-552. doi: 10.1093/oxfordjournals.molbev.a026334

Claverie, J. M. (2006). Viruses take center stage in cellular evolution. Genome Biol. 7:110. doi: 10.1186/gb-2006-7-6-110

Clerissi, C., Desdevises, Y., and Grimsley, N. (2012). Prasinoviruses of the marine green alga Ostreococcus tauri are mainly species specific. J. Virol. 86, 4611-4619. doi: 10.1128/JVI.07221-11

Colson, P., De Lamballerie, X., Yutin, N., Asgari, S., Bigot, Y., Bideshi, D. K., et al. (2013). "Megavirales," a proposed new order for eukaryotic nucleocytoplasmic large DNA viruses. Arch. Virol. 158, 2517-2521. doi: 10.1007/s00705-0131768-6 
Colson, P., Yutin, N., Shabalina, S. A., Robert, C., Fournous, G., La Scola, B., et al. (2011). Viruses with more than 1000 genes: Mamavirus, a new Acanthamoeba polyphaga mimivirus strain, and reannotation of Mimivirus genes. Genome Biol. Evol. 3, 737-742. doi: 10.1093/gbe/evr048

Couronne, O., Poliakov, A., Bray, N., Ishkhanov, T., Ryaboy, D., Rubin, E., et al. (2003). Strategies and tools for whole-genome alignments. Genome Res. 13, 73-80. doi: 10.1101/gr.762503

Darling, A. C., Mau, B., Blattner, F. R., and Perna, N. T. (2004). Mauve: multiple alignment of conserved genomic sequence with rearrangements. Genome Res. 14, 1394-1403. doi: 10.1101/gr.2289704

Desnues, C., La Scola, B., Yutin, N., Fournous, G., Robert, C., Azza, S., et al. (2012). Provirophages and transpovirons as the diverse mobilome of giant viruses. Proc. Natl. Acad. Sci. U.S.A. 109, 18078-18083. doi: 10.1073/pnas.1208835109

Dupuy, C., Periquet, G., Serbielle, C., Bezier, A., Louis, F., and Drezen, J. M. (2011). Transfer of a chromosomal Maverick to endogenous bracovirus in a parasitoid wasp. Genetica 139, 489-496. doi: 10.1007/s10709-011-9569-x

Edgar, R. C. (2004). MUSCLE: a multiple sequence alignment method with reduced time and space complexity. BMC Bioinformatics 5:113. doi: 10.1186/1471-21055-113

Elde, N. C., Child, S. J., Eickbush, M. T., Kitzman, J. O., Rogers, K. S., Shendure, J., et al. (2012). Poxviruses deploy genomic accordions to adapt rapidly against host antiviral defenses. Cell 150, 831-841. doi: 10.1016/j.cell.2012.05.049

Filee, J. (2013). Route of NCLDV evolution: the genomic accordion. Curr. Opin. Virol. 3, 595-599. doi: 10.1016/j.coviro.2013.07.003

Filee, J. (2014). Multiple occurrences of giant virus core genes acquired by eukaryotic genomes: the visible part of the iceberg? Virology 466-467, 53-59. doi: 10.1016/j.virol.2014.06.004

Filee, J., and Chandler, M. (2008). Convergent mechanisms of genome evolution of large and giant DNA viruses. Res. Microbiol. 159, 325-331. doi: 10.1016/j.resmic.2008.04.012

Filee, J., Pouget, N., and Chandler, M. (2008). Phylogenetic evidence for extensive lateral acquisition of cellular genes by Nucleocytoplasmic large DNA viruses. BMC Evol. Biol. 8:320. doi: 10.1186/1471-2148-8-320

Filee, J., Siguier, P., and Chandler, M. (2007). I am what I eat and I eat what I am: acquisition of bacterial genes by giant viruses. Trends Genet. 23, 10-15. doi: 10.1016/j.tig.2006.11.002

Forterre, P. (2010). Giant viruses: conflicts in revisiting the virus concept. Intervirology 53, 362-378. doi: 10.1159/000312921

Gilbert, C., Chateigner, A., Ernenwein, L., Barbe, V., Bezier, A., Herniou, E. A., et al. (2014). Population genomics supports baculoviruses as vectors of horizontal transfer of insect transposons. Nat. Commun. 5, 3348. doi: $10.1038 /$ ncomms 4348

Grasis, J. A., Lachnit, T., Anton-Erxleben, F., Lim, Y. W., Schmieder, R., Fraune, S., et al. (2014). Species-specific viromes in the ancestral holobiont Hydra. PLoS ONE 9:e109952. doi: 10.1371/journal.pone.0109952

Guindon, S., Delsuc, F., Dufayard, J. F., and Gascuel, O. (2009). Estimating maximum likelihood phylogenies with PhyML. Methods Mol. Biol. 537, 113-137. doi: 10.1007/978-1-59745-251-9_6

Iyer, L. M., Balaji, S., Koonin, E. V., and Aravind, L. (2006). Evolutionary genomics of nucleo-cytoplasmic large DNA viruses. Virus Res. 117, 156-184. doi: 10.1016/j.virusres.2006.01.009

Jeanniard, A., Dunigan, D. D., Gurnon, J. R., Agarkova, I. V., Kang, M., Vitek, J., et al. (2013). Towards defining the chloroviruses: a genomic journey through a genus of large DNA viruses. BMC Genomics 14:158. doi: 10.1186/1471-216414-158

Kobayashi, I. (2001). Behavior of restriction-modification systems as selfish mobile elements and their impact on genome evolution. Nucleic Acids Res. 29, 3742-3756. doi: 10.1093/nar/29.18.3742

Krupovic, M., and Koonin, E. V. (2015). Polintons: a hotbed of eukaryotic virus, transposon and plasmid evolution. Nat. Rev. Microbiol. 13, 105-115. doi: $10.1038 /$ nrmicro3389

Kutikhin, A. G., Yuzhalin, A. E., and Brusina, E. B. (2014). Mimiviridae, Marseilleviridae, and virophages as emerging human pathogens causing healthcare-associated infections. GMS Hyg. Infect. Control. 9, Doc16. doi: $10.3205 / \mathrm{dgkh} 000236$
Landstein, D., Burbank, D. E., Nietfeldt, J. W., and Van Etten, J. L. (1995). Large deletions in antigenic variants of the chlorella virus PBCV-1. Virology 214, 413-420. doi: 10.1006/viro.1995.0051

Lee, A. M., Ivey, R. G., Henry, E. C., and Meints, R. H. (1995). Characterization of a repetitive DNA element in a brown algal virus. Virology 212, 474-480. doi: 10.1006/viro.1995.1505

Maumus, F., Epert, A., Nogue, F., and Blanc, G. (2014). Plant genomes enclose footprints of past infections by giant virus relatives. Nat. Commun. 5, 4268. doi: $10.1038 /$ ncomms 5268

Moniruzzaman, M., Lecleir, G. R., Brown, C. M., Gobler, C. J., Bidle, K. D., Wilson, W. H., et al. (2014). Genome of brown tide virus (AaV), the little giant of the Megaviridae, elucidates NCLDV genome expansion and host-virus coevolution. Virology 466-467, 60-70. doi: 10.1016/j.virol.2014.06.031

Moreira, D., and Brochier-Armanet, C. (2008). Giant viruses, giant chimeras: the multiple evolutionary histories of Mimivirus genes. BMC Evol. Biol. 8:12. doi: 10.1186/1471-2148-8-12

Moreira, D., and Lopez-Garcia, P. (2005). Comment on "The 1.2 megabase genome sequence of Mimivirus." Science 308, 1114. doi: 10.1126/science. 1110820

Nishida, K., Kimura, Y., Kawasaki, T., Fujie, M., and Yamada, T. (1999). Genetic variation of chlorella viruses: variable regions localized on the CVK2 genomic DNA. Virology 255, 376-384. doi: 10.1006/viro.1998.9595

Philippe, N., Legendre, M., Doutre, G., Coute, Y., Poirot, O., Lescot, M., et al. (2013). Pandoraviruses: amoeba viruses with genomes up to $2.5 \mathrm{Mb}$ reaching that of parasitic eukaryotes. Science 341, 281-286. doi: 10.1126/science.1239181

Raoult, D., and Boyer, M. (2010). Amoebae as genitors and reservoirs of giant viruses. Intervirology 53, 321-329. doi: 10.1159/000312917

Suhre, K. (2005). Gene and genome duplication in Acanthamoeba polyphaga Mimivirus. J. Virol. 79, 14095-14101. doi: 10.1128/JVI.79.22.14095-14101.2005

Van Etten, J. L. (2003). Unusual life style of giant chlorella viruses. Annu. Rev. Genet. 37, 153-195. doi: 10.1146/annurev.genet.37.110801.143915

Yolken, R. H., Jones-Brando, L., Dunigan, D. D., Kannan, G., Dickerson, F., Severance, E., et al. (2014). Chlorovirus ATCV-1 is part of the human oropharyngeal virome and is associated with changes in cognitive functions in humans and mice. Proc. Natl. Acad. Sci. U.S.A. 111, 16106-16111. doi: 10.1073/pnas.1418895111

Yoosuf, N., Yutin, N., Colson, P., Shabalina, S. A., Pagnier, I., Robert, C., et al. (2012). Related giant viruses in distant locations and different habitats: Acanthamoeba polyphaga moumouvirus represents a third lineage of the Mimiviridae that is close to the megavirus lineage. Genome Biol. Evol. 4, 1324-1330. doi: 10.1093/gbe/evs109

Yutin, N., Colson, P., Raoult, D., and Koonin, E. V. (2013). Mimiviridae: clusters of orthologous genes, reconstruction of gene repertoire evolution and proposed expansion of the giant virus family. Virol. J. 10, 106. doi: 10.1186/1743-422X10-106

Yutin, N., and Koonin, E. V. (2013). Pandoraviruses are highly derived phycodnaviruses. Biol. Direct 8:25. doi: 10.1186/1745-6150-8-25

Yutin, N., Wolf, Y. I., and Koonin, E. V. (2014). Origin of giant viruses from smaller DNA viruses not from a fourth domain of cellular life. Virology 466-467, 38-52. doi: 10.1016/j.virol.2014.06.032

Yutin, N., Wolf, Y. I., Raoult, D., and Koonin, E. V. (2009). Eukaryotic large nucleo-cytoplasmic DNA viruses: clusters of orthologous genes and reconstruction of viral genome evolution. Virol. J. 6:223. doi: 10.1186/1743$422 \mathrm{X}-6-223$

Conflict of Interest Statement: The author declares that the research was conducted in the absence of any commercial or financial relationships that could be construed as a potential conflict of interest.

Copyright (C) 2015 Filée. This is an open-access article distributed under the terms of the Creative Commons Attribution License (CC BY). The use, distribution or reproduction in other forums is permitted, provided the original author(s) or licensor are credited and that the original publication in this journal is cited, in accordance with accepted academic practice. No use, distribution or reproduction is permitted which does not comply with these terms. 